\title{
Knots with identical Khovanov homology
}

\author{
LIAM WATSON
}

\begin{abstract}
We give a recipe for constructing families of distinct knots that have identical Khovanov homology and give examples of pairs of prime knots, as well as infinite families, with this property.
\end{abstract}

57M25, 57M27

\section{Introduction}

Khovanov homology [7] is an invariant which associates a bi-graded abelian group to a knot (or link) in $S^{3}$. The Jones polynomial of the knot arises as a graded Euler characteristic of this theory, and as such questions about the Jones polynomial may be rephrased in terms of Khovanov homology. It is unknown for example, if either of these invariants detects the unknot. On the other hand, Khovanov homology is known to be strictly stronger than the Jones polyniomial: Bar-Natan provided examples of knots with the same Jones polynomial that are distinguished by Khovanov homology [2]. There have been many techniques developed for producing pairs of knots that have the same Jones polynomial, and it is natural to ask if these techniques also preserve Khovanov homology. One of the simplest techniques for generating distinct knots with the same Jones polynomial is mutation, however it is currently unknown if mutation of knots leaves Khovanov homology invariant (see Bar-Natan [1] and Wehrli [19]).

The aim of this note is to present a construction giving rise to distinct knots that cannot be distinguished using Khovanov homology. Our main tool is the long exact sequence in Khovanov homology which is presented, along with a review of Khovanov homology, in Section 2. In Section 3 we present a general construction for producing pairs of knots with identical Khovanov homology. This construction is applied in Section 4 to obtain pairs of distinct prime knots with identical Khovanov homology (Theorem 4.1). These examples are distinguished by the HOMFLYPT polynomial, and as such must have distinct triply-graded link homology (see Khovanov [6] and KhovanovRozansky [8]). In Section 5 we give infinite families of distinct knots with identical Khovanov homology (Theorem 5.1), and in Section 6 we construct an infinite family of knots admitting a mutation that is not detected by Khovanov homology (Proposition 6.1). We conclude with a series of examples in Section 7. 


\section{Background and notation}

We briefly review Khovanov homology to solidify notation, and refer the reader to Khovanov's original paper [7], as well as the work of Bar-Natan [2; 3] and Rasmussen [13].

The Khovanov complex of a knot $K$ is generated by first considering an $n$-crossing diagram for $K$ together with $2^{n}$ states, each of which is a collection of disjoint simple closed curves in the plane. Each state $s$ is obtained from a choice of resolution $\asymp$ (the 0 -resolution) or ) ( (the 1-resolution) for every crossing $\mathcal{X}$. By fixing an order on the crossings, each state $s$ may be represented by an $n$-tuple with entries in $\{0,1\}$ so that the states may be arranged at the vertices of the $n$-cube $[0,1]^{n}$ (the cube of resolutions for $K$ ). Let $|s|$ be the sum of the entries of the $n$-tuple associated to $s$ (the height of $s$ ).

Let $V$ be a free, graded $\mathbb{Z}$-module generated by $\left\langle v_{-}, v_{+}\right\rangle$, where $\operatorname{deg}\left(v_{ \pm}\right)= \pm 1$. To each state we associate $V^{\otimes \ell_{s}}$ where $\ell_{s}>0$ is the number of closed curves in the given state. The associated grading is referred to as the Jones grading, denoted by $q$. Set

$$
\mathcal{C}^{u}(K)=\bigoplus_{u=|s|} V^{\otimes \ell_{s}}\{|s|\}
$$

where $\{\cdot\}$ shifts the Jones grading via $(W\{j\})_{q}=W_{q-j}$. The chain groups of the Khovanov complex are given by

$$
\mathrm{CKh}_{q}^{u}(K)=\left(\mathcal{C}(K)\left[-n_{-}\right]\left\{n_{+}-2 n_{-}\right\}\right)_{q}^{u}=\mathcal{C}_{q-n_{+}+2 n_{-}}^{u+n_{-}}(K)
$$

where [.] shifts the homological grading $u$ as shown. For a given orientation of $K$, $n_{+}=n_{+}(K)$ is the number of positive crossings $K$ in $K$ and $n_{-}=n_{-}(K)$ is the number of negative crossings $\bigwedge^{\pi}$ in $K$.

The differentials $\partial^{u}: \mathrm{CKh}^{u}(K) \rightarrow \mathrm{CKh}^{u+1}(K)$ come from the collection of edges in the cube of resolutions moving from height $u$ to height $u+1$. Each of these edges corresponds to exactly one of two operations $(m: V \otimes V \rightarrow V$ and $\Delta: V \rightarrow V \otimes V)$ of a Frobenius algebra defined over $V$, since each edge can be identified with exactly one change of the form $\asymp \rightarrow$ ) ( or ) $(\rightarrow \asymp$. Fixing a convention so that the faces of the cube anti-commute, $\partial^{u}$ is the sum of all the maps at the prescribed height. The Khovanov homology $\mathrm{Kh}(K)$, defined as the homology of the complex $\left(\mathrm{CKh}^{u}(K), \partial^{u}\right)$, is an invariant of the knot $K$; the (unnormalized) Jones polynomial of $K$ arises as

$$
\sum_{j} \sum_{u}(-1)^{u} q^{j} \operatorname{dim}\left(\mathrm{Kh}_{j}^{u}(K) \otimes \mathbb{Q}\right) .
$$

Algebraic $\mathcal{E}$ Geometric Topology, Volume 7 (2007) 
Given a knot $K(\nwarrow)$ with a distinguished positive crossing, there is a short exact sequence

$$
0 \longrightarrow \mathcal{C}(K(\asymp))[1]\{1\} \longrightarrow \mathcal{C}(K(\text { 的) } \rightarrow \mathcal{C}(K()()) \longrightarrow 0 .
$$

Since $K()()$ inherits the orientation of $K(\nearrow)$, we set $c=n_{-}(K(\asymp))-n_{-}(K(\nwarrow))$ for some choice of orientation on $K(\asymp)$ to obtain

$$
0 \longrightarrow \mathrm{CKh}_{q-3 c-2}^{u-c-1}(K(\asymp)) \longrightarrow \mathrm{CKh}_{q}^{u}\left(K(\text { 年) }) \longrightarrow \mathrm{CKh}_{q-1}^{u}(K()()) \longrightarrow 0 .\right.
$$

This short exact sequence gives rise to a long exact sequence

$$
\rightarrow \mathrm{Kh}_{q-3 c-2}^{u-c-1}(K(\asymp)) \rightarrow \mathrm{Kh}_{q}^{u}\left(K(\text { 以) }) \rightarrow \mathrm{Kh}_{q-1}^{u}(K()()) \stackrel{\delta_{*}}{\rightarrow} \mathrm{Kh}_{q-3 c-2}^{u-c}(K(\asymp)) \rightarrow\right.
$$

where $\delta_{*}$ is the map induced on homology from (the component of) the differential $\delta: \mathrm{CKh}_{q-1}^{u}(K()()) \rightarrow \mathrm{CKh}_{q-3 c-2}^{u-c-1}(K(\asymp))$.

Similarly, for a knot $K\left(\lambda^{\pi}\right)$ with a distinguished negative crossing there is a long exact sequence:

$$
\rightarrow \mathrm{Kh}_{q+1}^{u}(K()()) \rightarrow \mathrm{Kh}_{q}^{u}\left(K\left(\nearrow^{\prime}\right)\right) \rightarrow \mathrm{Kh}_{q-3 c-1}^{u-c}(K(\smile)) \stackrel{\delta_{*}}{\rightarrow} \mathrm{Kh}_{q+1}^{u+1}(K()()) \rightarrow .
$$

We will make use of one further piece of structure on $\mathrm{Kh}(K)$ introduced by Lee [9] and Rasmussen [12]. The bigraded abelian group $E_{1}^{u, q}=\mathrm{Kh}_{q}^{u}(K) \otimes \mathbb{Q}$ associated to a knot $K$ may be viewed as the first sheet of a spectral sequence (the Lee spectral sequence) with differential on the $E_{i}$ sheet of bidegree $(1,4 i)$. In particular, each of the sums

and

$$
\begin{gathered}
\sum_{q \equiv 1(\bmod 4)} \sum_{u}(-1)^{u} \operatorname{dim}\left(E_{i}^{u, q}\right) \\
\sum_{q \equiv-1(\bmod 4)} \sum_{u}(-1)^{u} \operatorname{dim}\left(E_{i}^{u, q}\right)
\end{gathered}
$$

must be constant for all $i$. Further, the spectral sequence converges to $E_{\infty}^{u, q} \cong \mathbb{Q} \oplus \mathbb{Q}$ with $E_{\infty}^{u, s \pm 1} \cong \mathbb{Q}$, where the even integer $s$ is Rasmussen's invariant. As a result the above constant is 1 in both cases, giving rise to:

$$
\begin{gathered}
\sum_{q \equiv 1(\bmod 4)} \sum_{u}(-1)^{u} \operatorname{dim}\left(\mathrm{Kh}_{q}^{u}(K) \otimes \mathbb{Q}\right)=1 \\
\sum_{q \equiv-1(\bmod 4)} \sum_{u}(-1)^{u} \operatorname{dim}\left(\mathrm{Kh}_{q}^{u}(K) \otimes \mathbb{Q}\right)=1 .
\end{gathered}
$$

Algebraic 83 Geometric Topology, Volume 7 (2007) 


\section{Construction}

Consider the knot $K=K_{\beta}(T, U)$ shown in Figure 1 where $\beta$ is an element of the three strand braid group $B_{3}$ with inverse $\beta^{-1} . T$ and $U$ are tangles (or Conway tangles), that is $T=\left(B_{T}^{3}, \tau\right)$ and $U=\left(B_{U}^{3}, \mu\right)$ where $B_{T}^{3}$ (respectively $B_{U}^{3}$ ) is a 3-ball containing a collection of arcs $\tau$ (respectively $\mu$ ) that intersect the boundary of the 3-ball transversally in exactly 4 points (see Lickorish [10] and Rolfsen [15]).

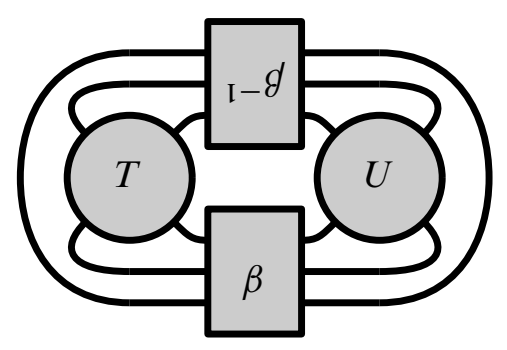

Figure 1: The knot $K_{\beta}(T, U)$.

There is a well defined $\mathbb{Z}$-action (a half-twist action) on the set of isotopy classes (fixing endpoints) of tangles that comes from the two strand braid group. For a given tangle $T$, write $T^{\sigma}=T \backslash$ and $T^{\bar{\sigma}}=T\left(T /\right.$ where $\langle\sigma\rangle=\mathbb{Z} \cong B_{2}$ and $\sigma \bar{\sigma}=e$ (that is, $\sigma=\backslash$ is the standard braid generator). Let $K^{\sigma}=K_{\beta}\left(T^{\sigma}, U^{\bar{\sigma}}\right)$.

The sum $T+U$ of two tangles is defined by side-by-side concatenation. This generalizes the half-twist-action: $T^{\sigma}$ may be denoted $T+\bigotimes(\sigma$ adds a twist). A tangle $T$ is called simple if $T+\bigotimes$ is isotopic (fixing endpoints) to 0 . Note that $T^{\sigma}$ is simple if and only if $T$ is simple. Also, if $T$ and $U$ are simple, then so is the tangle $T+U$

For the purposes of this paper, assume that the tangles considered have no closed components (that is, $\tau$ and $\mu$ are each a pair of arcs). Note also that since we are considering knots, we may restrict attention to tangles $T$ and $U$ that have connectivity of the form $\bigotimes$ and $\bigotimes$ (tangles having connectivity $\bigotimes$ generally give rise to links, moreover such tangles are never simple). Further, we assume throughout that the braid $\beta \in B_{3}$ is such that $K=K_{\beta}(T, U)$ has only one component.

Lemma 3.1 For simple tangles $T, U$ the knots $K=K_{\beta}(T, U)$ and $K^{\sigma}=K_{\beta}\left(T^{\sigma}, U^{\bar{\sigma}}\right)$ have identical Khovanov homology.

Remark We could also consider a similar action that adds twists to tangles on the left; we make use of this in Section 6. 
Remark It follows from Lemma 3.1 that the knots $K$ and $K^{\sigma}$ have identical Jones polynomial. This fact is proved by the author [18, Lemma 5.2] without the requirement that the tangles be simple.

Proof of Lemma 3.1 The proof is an application of the long exact sequences introduced in Section 2. The reader may find it useful to follow the general argument through on a particular example such as that of Section 7.1.

Our strategy is to distinguish two crossings of $K^{\sigma}=K_{\beta}\left(T^{\sigma}, U^{\bar{\sigma}}\right)$ (the two crossings added by the action of $\sigma)$ and write $K(\searrow$ $\)=K_{\beta}\left(T^{\sigma}, U^{\bar{\sigma}}\right)$ so that $K(\asymp こ)=$ $K_{\beta}(T, U)$. Since the tangles $T$ and $U$ are simple by hypothesis, it is easy to check that $K()($ () and $K(\asymp)()$ (as well as $K(\searrow)()$ and $K()(こ)$ ) are diagrams for the 2 -component trivial link (apply simplicity, and note that the braids are allowed to cancel). Let

$$
L=\mathrm{Kh}(\bigcirc \bigcirc)=V \otimes V \cong(\mathbb{Z})_{-2} \oplus(\mathbb{Z} \oplus \mathbb{Z})_{0} \oplus(\mathbb{Z})_{2}
$$

and note that $L^{u}=0$ in all homological degrees $u \neq 0$.

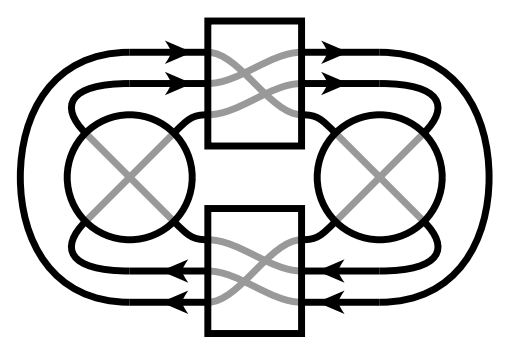

Figure 2: An orientation for the knot $K^{\sigma}$ when the permutation associated to $\beta$ is $\left(\begin{array}{lll}1 & 3 & 2\end{array}\right)$.

Since it will be necessary to fix orientations, we divide into six cases according to the various possible permutations associated to braids in $B_{3}$. First suppose that the permutation associated to $\beta$ is ( $\left.\begin{array}{lll}1 & 3 & 2\end{array}\right)$ (as is the case for $\beta=\sigma_{1}^{-1} \sigma_{2} \sigma_{1}^{-2 n}$, for example).

Now suppose that both $T^{\sigma}$ and $U^{\bar{\sigma}}$ have connectivity of the form $\bigotimes$. Then it is easy to check that $T$ and $U$ have connectivity of the form $Q$. Moreover, since the permutation associated to $\beta$ is $\left(\begin{array}{lll}1 & 3 & 2\end{array}\right)$, the permutation associated to $\beta^{-1}$ is $\left(\begin{array}{lll}1 & 2 & 3\end{array}\right)$

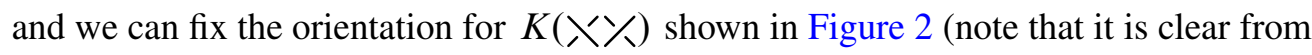
this diagram that the knot in question has one component). With this orientation in hand, we have that the distinguished crossing of $T^{\sigma}$ is negative, while the distinguished crossing of $U^{\bar{\sigma}}$ is positive: $K\left(\bigwedge_{\nwarrow}\right.$ ) $)$. Notice that the resolution $K(こ ソ)$ (that is, the 


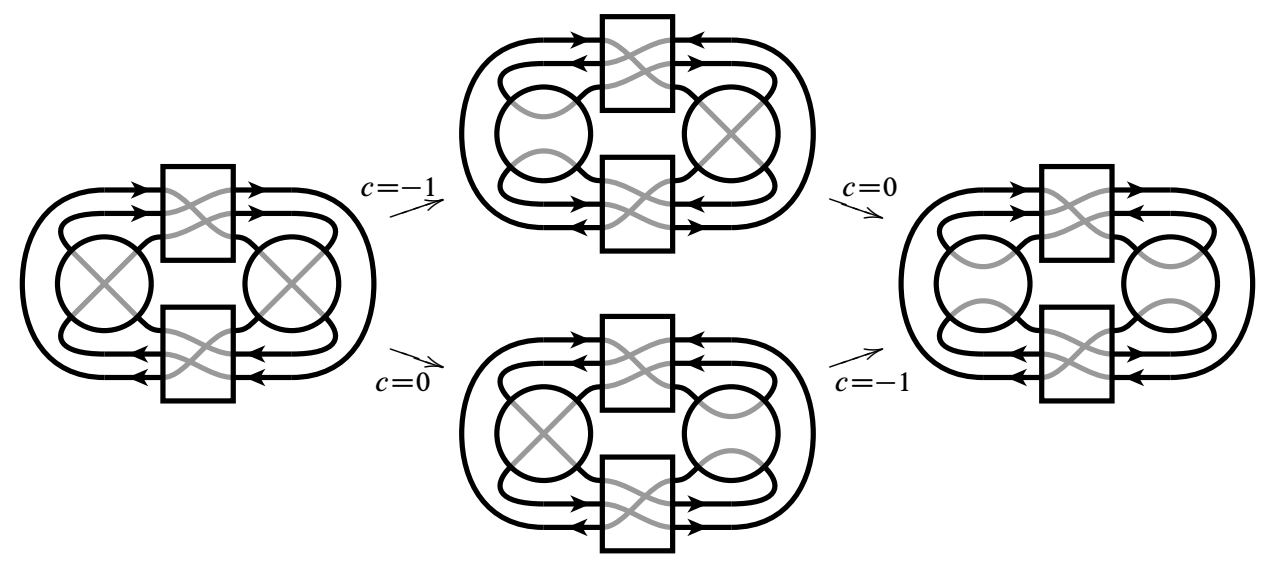

Figure 3: Values of $c$ upon resolution.

tangle $T$ ) does not inherit this orientation. If we resolve with respect to the left-most (distinguished) crossing, we have the exact sequence

$$
L_{q+1}^{u} \longrightarrow \mathrm{Kh}_{q}^{u} K(\text { メソ }) \longrightarrow \mathrm{Kh}_{q-3 c-1}^{u-c} K(\asymp \text { こ }) \longrightarrow L_{q+1}^{u+1}
$$

where one can check that $c=n_{-}(K(こ ソ))-n_{-}(K($ メソ $))=-1$.

Indeed, since the braids chosen are inverses of each other, the number of negative and positive crossings contributed by the braids remains constant. Therefore, to compute the values for $c$ we need only consider the tangles $T^{\sigma}$ and $U^{\bar{\sigma}}$. Upon resolution, notice that the orientation on $U^{\bar{\sigma}}$ is preserved (see Figure 3), while the new orientation for the resolution of $T^{\sigma}$ (that is, $T$ ) has precisely one less negative crossing (the crossing we resolved). This is because the new orientation reverses the orientation on both strands (it can be checked that this will always preserve the number of positive and negative crossings) so that

$$
\begin{aligned}
c & =n_{-}(K(こ ソ))_{-}(K(\text { メソ }) \\
& =n_{-}(T)-n_{-}\left(T^{\sigma}\right) \\
& \left.=n_{-}(2)^{\prime}\right)_{-}(2) \\
& =-1 .
\end{aligned}
$$


Therefore, the exact sequence is given by

$$
L_{q+1}^{u} \longrightarrow \mathrm{Kh}_{q}^{u} K(\text { メソ }) \longrightarrow \mathrm{Kh}_{q+2}^{u+1} K(\asymp \text { こ }) \longrightarrow L_{q+1}^{u+1} .
$$

Now, resolving the second crossing, we make similar observations. The orientation on the strands of the tangle $T$ are both reversed once more (see Figure 3), so that the number of negative crossings contributed by $T$ is left unchanged. On the other hand, the resolution taking $U^{\bar{\sigma}}$ to $U$ removes a positive crossing, and preserves the orientation on the tangle $U$. Therefore

$$
\begin{aligned}
c & =n_{-}(K(こ こ))-n_{-}(K(こ ソ)) \\
& =n_{-}(U)-n_{-}\left(U^{\bar{\sigma}}\right) \\
& =n_{-}(2) \\
& =0
\end{aligned}
$$

and we have the exact sequence

$$
L_{q-1}^{u-1} \longrightarrow \mathrm{Kh}_{q-2}^{u-1} K(\asymp こ) \longrightarrow \mathrm{Kh}_{q}^{u} K(こ ソ) \longrightarrow L_{q-1}^{u}
$$

which may be rewritten as

$$
L_{q+1}^{u} \longrightarrow \mathrm{Kh}_{q}^{u} K(こ こ) \longrightarrow \mathrm{Kh}_{q+2}^{u+1} K(こ ソ) \longrightarrow L_{q+1}^{u+1}
$$

Combining the exact sequences (3) and (4) we obtain the the diagram of exact sequences

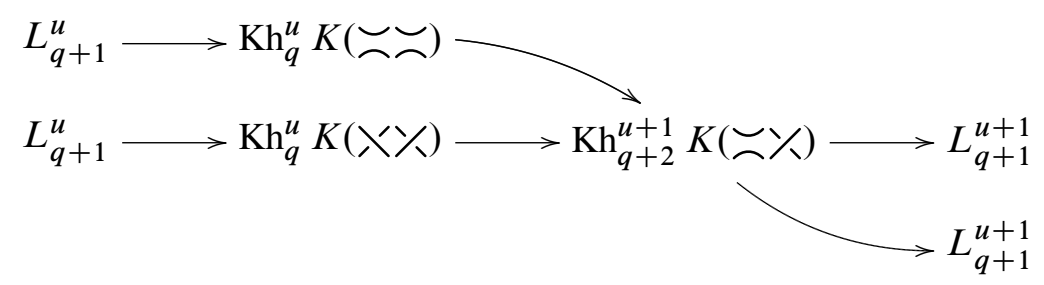

from which we deduce that

$$
\mathrm{Kh}_{q}^{u} K(\text { メソ }) \cong \mathrm{Kh}_{q+2}^{u+1} K(\asymp \aleph) \cong \mathrm{Kh}_{q}^{u} K(\asymp こ)
$$

Algebraic $8 \mathcal{G}$ Geometric Topology, Volume 7 (2007) 
for all homological gradings $u>0$ since $L^{u}=0$. Moreover, when $u=0$ we have

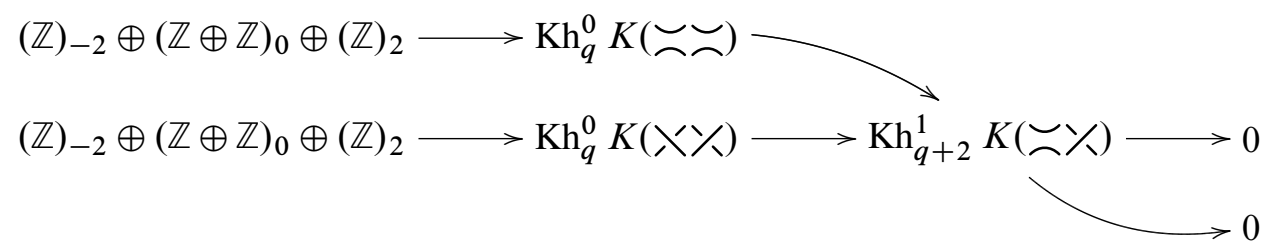

so that

$$
\mathrm{Kh}_{q}^{0} K(\text { メソ }) \cong \mathrm{Kh}_{q}^{0} K(こ こ)
$$

for $q \neq-3,-1,1$.

A slightly different diagram of exact sequences is obtained if the right-most distin-

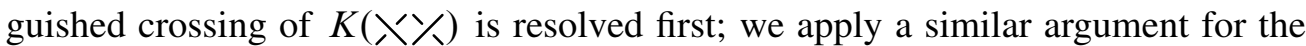
(switched) values of $c$. This time, we first resolve the (positive) crossing of $U^{\bar{\sigma}}$ to obtain $U$ with its orientation unchanged (see Figure 3 ). The induced orientation on $T^{\sigma}$ reverses the orientation of both strands (as before) so that the number of positive and negative crossings contributed by $T^{\sigma}$ are once more unchanged (of course, the contribution from the braids is constant, as before). Therefore, we lose only a positive crossing, and obtain

$$
\begin{aligned}
c & =n_{-}(K(\text { メ }))-n_{-}(K(\text { メイ })) \\
& =n_{-}(U)-n_{-}\left(U^{\bar{\sigma}}\right) \\
& =n_{-}(2) \\
& =0,
\end{aligned}
$$

which in turn gives the exact sequence

$$
L_{q-1}^{u-1} \longrightarrow \mathrm{Kh}_{q-2}^{u-1} K(\text { メこ }) \longrightarrow \mathrm{Kh}_{q}^{u} K(\text { メハ }) \longrightarrow L_{q-1}^{u}
$$

Resolving the distinguished crossing of $T^{\sigma}$ to obtain $T$, we have that the orientation on $U$ is once more preserved (see Figure 3), so that the contribution to $c$ comes from comparing $T$ and $T^{\sigma}$ only. Once again, we remove the distinguished crossing (a 
negative crossing) and reverse orientation of both the strands of $T$. Therefore,

$$
\begin{aligned}
c & =n_{-}(K(\asymp こ))-n_{-}(K(\text { メこ }) \\
& =n_{-}(T)-n_{-}\left(T^{\sigma}\right) \\
& =n_{-}(2 / 2)-n_{-}(2) \\
& =-1
\end{aligned}
$$

and then we obtain the sequence

$$
L_{q-1}^{u-1} \longrightarrow \mathrm{Kh}_{q-2}^{u-1} K(\nearrow) \longrightarrow \mathrm{Kh}_{q}^{u} K(こ こ) \longrightarrow L_{q-1}^{u}
$$

where the gradings are shifted accordingly as in the case of the exact sequence (4). This time, sequences (6) and (7) combine to give the diagram of exact sequences

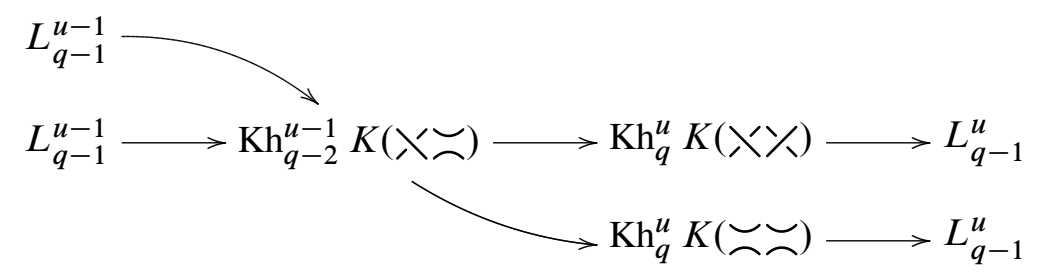

and we obtain the isomorphism

$$
\mathrm{Kh}_{q}^{u} K(\text { メソ }) \cong \mathrm{Kh}_{q-2}^{u-1} K(\text { こ }) \cong \mathrm{Kh}_{q}^{u} K(こ こ)
$$

whenever $u<0$. When $u=0$, we have

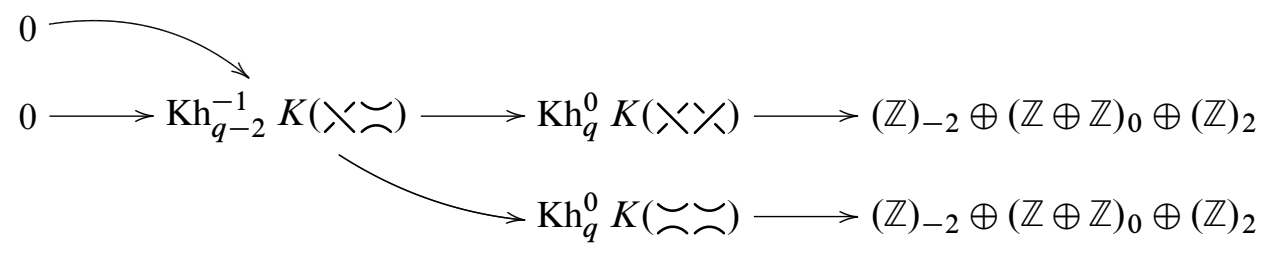

so that

$$
\mathrm{Kh}_{q}^{0} K(\text { メソ }) \cong \mathrm{Kh}_{q}^{0} K(こ こ)
$$

for $q \neq-1,1,3$.

Combining the information from diagrams (5) and (8), we conclude that

$$
\mathrm{Kh}_{q}^{u} K(\text { メソ }) \cong \mathrm{Kh}_{q}^{u} K(こ こ)
$$

Algebraic $8 \mathcal{G}$ Geometric Topology, Volume 7 (2007) 
except when $u=0$ and $q= \pm 1$. In fact, diagram (8) tells us that the torsion parts for $u=0$ and $q= \pm 1$ are isomorphic. Indeed, since $L$ is torsion free we have

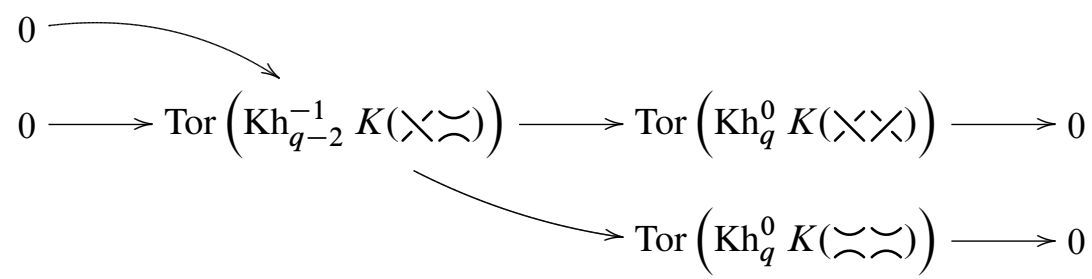

hence

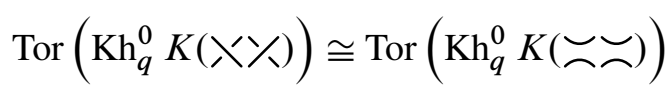

for all $q$.

We pause here to remark that the cases with different connectivity for $T^{\sigma}$ and $U^{\bar{\sigma}}$ $(Q, Q$ and $Q, Q$ and $Q, 8)$ proceed in the same way, with only minor adjustments to the induced orientations. In fact, the proof amounts to reordering and/or rotating the oriented diagrams encountered in Figure 3. We leave this step to the reader.

To treat the other possible permutations associated to $\beta$ it suffices to check that the same values for $c$ are obtained upon resolution of the distinguished crossings. If this is the case, then the rest of the argument goes through unchanged. First notice that the if the permutation associated to $\beta$ is $\left(\begin{array}{lll}1 & 2 & 3\end{array}\right)$ then it suffices to rotate each of the diagrams in Figure 3 by 180 degrees and exchange the diagrams of the middle column to obtain the same values for $c$. We do not need to consider the permutations (1) or (23) since it can be checked that if $\beta$ has either of these associated permutations, the knot $K_{\beta}(T, U)$ will have more than one component. It remains to check the permutations

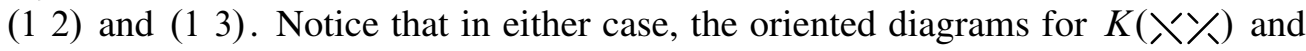
$K(こ こ)$ are exactly as in Figure 3 (with obvious adjustments to the connectivity of the

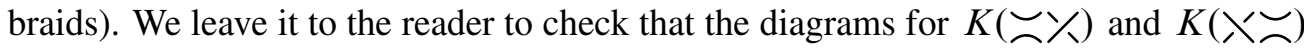
in both cases admit orientations that give rise to:

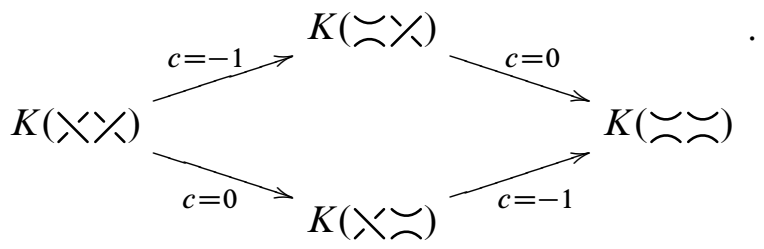

This in turn gives rise to the same diagrams of exact sequences (5) and (8) for all $\beta$ such that $K_{\beta}(T, U)$ has one component. 
Finally, note that acting by $\bar{\sigma}$ (instead of $\sigma$ ) on $K$ switches the two exact sequences in each of the diagrams (5) and (8). This is due once more to the values obtained for $\mathrm{c}$ :

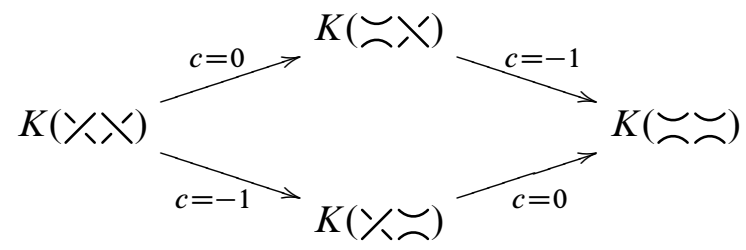

In particular, we obtain the same isomorphisms of Khovanov homology groups.

With these observations in hand, it remains now to analyze the the free part of $\mathrm{Kh}_{ \pm 1}^{0} K($ メソ $)$ and $\mathrm{Kh}_{ \pm 1}^{0}(こ こ)$. To this end we work over $\mathbb{Q}$, and apply Equation (1) and Equation (2).

Suppose $q=1$, then from Equation (1) we have that

$$
\begin{aligned}
\sum_{q \equiv 1(\bmod 4)} \sum_{u}(-1)^{u} \operatorname{dim}\left(\operatorname{Kh}_{q}^{u} K(\nearrow \text { ソ }) \otimes \mathbb{Q}\right) & \\
= & \sum_{q \equiv 1(\bmod 4)} \sum_{u}(-1)^{u} \operatorname{dim}\left(\operatorname{Kh}_{q}^{u} K(こ こ) \otimes \mathbb{Q}\right)
\end{aligned}
$$

and since all groups are isomorphic away from $(u, q)=(0, \pm 1)$, this implies that

$$
\operatorname{dim}\left(\mathrm{Kh}_{1}^{0} K(\nearrow \text { K }) \otimes \mathbb{Q}\right)=\operatorname{dim}\left(\mathrm{Kh}_{1}^{0} K(こ こ) \otimes \mathbb{Q}\right) .
$$

In particular, $\mathrm{Kh}_{1}^{0} K($ メソ $) \otimes \mathbb{Q} \cong \mathrm{Kh}_{1}^{0} K(こ こ) \otimes \mathbb{Q}$.

Applying a similar argument to the case $q=-1$ using Equation (2) gives the required isomorphism $\mathrm{Kh}_{-1}^{0} K(\backslash \backslash) \otimes \mathbb{Q} \cong \mathrm{Kh}_{-1}^{0} K(こ こ) \otimes \mathbb{Q}$ and we conclude that

$$
\operatorname{Kh}(K) \cong \operatorname{Kh}\left(K^{\sigma}\right) \text {. }
$$

We have yet to see that the knots $K$ and $K^{\sigma}$ are distinct. This is the focus of Section 4, Section 5 and Section 6; examples are given in Section 7.

\section{Distinct prime knots with identical Khovanov homology}

According to Lickorish [10, Theorem 5], a tangle $T=\left(B_{T}^{3}, \tau\right)$ is prime if and only if the two-fold branched cover of $B_{T}^{3}$ (branched over $\tau$ ) is irreducible and boundary irreducible. Since the two-fold cover of a sphere with 4 branch points is a torus, prime tangles are those two-fold branched covered by non-trivial knot complements. Note that $T$ is prime if and only if $T^{\sigma}$ is prime. 
Theorem 4.1 For every simple, prime tangle $T$ there exists a pair of distinct prime knots (each containing $T$ ) with identical Khovanov homology but distinct HOMFLYPT polynomial (and hence distinct triply-graded link homology).

Proof Choose $\beta=\sigma_{1}^{-1} \sigma_{2} \sigma_{1}^{-2}$ and the pair of tangles $\left(T, T^{\star}\right)$ in the configuration of Figure 1 where $U=T^{\star}$ is the mirror image of $T$ (hence prime and simple). This gives rise to a pair of knots $K_{\beta}\left(T, T^{\star}\right)$ and $K_{\beta}\left(T^{\sigma},\left(T^{\star}\right)^{\bar{\sigma}}\right)$ with identical Khovanov homology by applying Lemma 3.1. It was show by the author [18, Theorem 1.1] that this pair of knots have distinct HOMFLYPT polynomials from which we conclude that the knots are distinct, and observe that they must have different triply-graded homology. Finally, it follows from the work of Lickorish [10, Theorem 1 and Lemma 2] that both of the knots constructed are prime whenever $T$ is a prime tangle; a complete argument is given in [18, Theorem 1.1].

Remark Since the pair of knots generated in the proof of Theorem 4.1 have distinct HOMFLYPT polynomial they can not be related by mutation (cf Section 6).

Examples of knots arising as in Theorem 4.1 (in particular, examples of prime simple tangles) are given in Section 7.2.

\section{Constructing infinite families}

Although one needs a mechanism to prove that the knots obtained are distinct, the action of $\sigma$ defined in Section 3 may be iterated to obtain infinite families of knots with identical Khovanov homology. Luse and Rong [11] classified the particular family $K_{\beta}(T, U)$ taking $\beta=\sigma_{1}^{-1} \sigma_{2} \sigma_{1}^{-2 n}$ in the case where $T$ and $U$ horizontal full-twists.

Theorem 5.1 For each $n \in \mathbb{N}$ there is an infinite family of distinct knots with identical Khovanov homology.

Proof Fix $n \in \mathbb{N}$ and consider the family of knots $K_{\ell}=K_{\beta}\left(\sigma^{2 \ell}, \sigma^{-2 \ell}\right)$ where $\sigma^{2 \ell}$ is the tangle consisting of $\ell$ horizontal full-twists, and $\beta=\sigma_{1}^{-1} \sigma_{2} \sigma_{1}^{-2 n}$. The tangles are clearly simple, so by iterating Lemma $3.1 K_{\ell}$ and $K_{\ell^{\prime}}$ have identical Khovanov homology for any $\ell, \ell^{\prime} \in \mathbb{Z}$. According to [11, Theorem 1.1], $K_{\ell}$ and $K_{\ell^{\prime}}$ are distinct knots whenever $\operatorname{gcd}(\ell, 2 n+1) \neq \operatorname{gcd}\left(\ell^{\prime}, 2 n+1\right)$. If $p_{1}^{\alpha_{1}} p_{2}^{\alpha_{2}} \cdots p_{k}^{\alpha_{k}}$ is the prime decomposition of $2 n+1$, we can choose $\ell=p_{i}$ (for any of the $i \in\{1, \ldots, k\}$ ) so that $\operatorname{gcd}(\ell, 2 n+1)=p_{i}$. Letting $\ell^{\prime}$ range over all primes that do not appear in the prime decomposition of $2 n+1$ gives the result.

Remark The classification of this family of knots in the case $n=1$ is due to Kanenobu [5]; this example is given in Section 7.4. 


\section{A remark on mutation}

Knot mutation (cf Rolfsen [15]) is a well known operation on tangles that alters knots without changing any of the skein-type polynomial invariants (ie Jones, HOMFLYPT, ... ). Although Wehrli has given examples of split links related by mutation that have different Khovanov homology [19], it is unknown if mutation preserves Khovanov homology for knots $[1 ; 19]$. As a third application of Lemma 3.1, we give an infinite family of knots that admit a mutation which is not detected by Khovanov homology.

Consider the family of knots $K_{m}(T)=K_{\beta}\left(\sigma^{m}, T\right)$ as in Figure 1 , where $\sigma^{m}$ is the tangle consisting of $m \in \mathbb{Z}$ horizontal half-twists (and $\beta$ is such that $K_{m}(T)$ has only one component).

Proposition 6.1 The mutation $\mu$ that flips a simple tangle $T$ in the knot digram $K_{m}(T)$ across the horizontal axis is not detected by Khovanov homology. That is, $\mathrm{Kh}\left(K_{m}(T)\right) \cong \mathrm{Kh}\left(K_{m}(\mu T)\right)$ for all $m \in \mathbb{Z}$.

Proof If we consider a similar construction to that of Section 3 allowing $B_{2}$ to act on the left (ie $\sigma: T \mapsto \lambda(T)$ and $\bar{\sigma}: T \mapsto Y(T)$ ), the proof of Lemma 3.1 goes through in the same way for this left action (on the same class of knots), and hence leaves Khovanov homology invariant. Indeed, one need only consider a 180 degree rotation of Figure 1, and the proof goes through verbatim after renaming $\beta=\beta^{-1}$, $T=\Omega$ and $U=L$.

Since the tangles $T$ and $\sigma^{m}$ are simple, we can can apply Lemma 3.1 to $K_{m}(T)$ resulting in a new knot $K_{m+1}\left(T^{\bar{\sigma}}\right)$ with identical Khovanov homology (note that with this notation $\left.\left(\sigma^{m}\right)^{\sigma}=\sigma^{m+1}\right)$. The key observation is that acting on the left by the inverse $\bar{\sigma}$ removes the twist added to $\sigma^{m}$, while the result of acting by $\sigma$ on the left of $T^{\bar{\sigma}}$ is a tangle isotopic (fixing endpoints) to the mutant $\mu T$. Hence we obtain a third knot $K_{m}(\mu T)$ - precisely the desired mutant - with identical Khovanov homology.

The elements of the proof of Proposition 6.1 can be seen in the example given in Section 7.5, in particular Figure 10.

\section{Examples}

The preceding sections show that the construction of Section 3 gives rise to a wide range of knots; in this section we give some particular examples. The notation for knots used below is consistent with Rolfsen's notation [14] for knots with fewer that 11 crossings, and KNOTSCAPE notation [17] otherwise up to mirrors (see also the Knot Atlas [4]). 


\subsection{A first example}
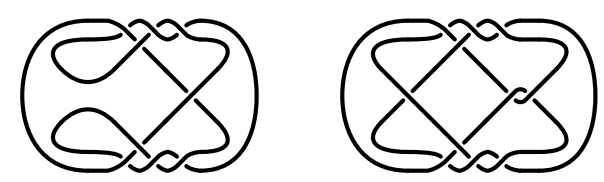

Figure 4: The knots $8_{8}$ and $10_{129}$.

The knots $8_{8}$ and $10_{129}$ admit diagrams of the form of Figure 1 with $\beta=\sigma_{1}^{-1} \sigma_{2} \sigma_{1}^{-2}$, as shown in Figure 4. This explains the coincidence in Khovanov homology enjoyed by this pair of knots, a fact well documented in [4]. It may be illustrative to revisit the proof of Lemma 3.1 with this particular example in hand. Observe that as a result of this choice of diagrams, $8_{8}$ is obtained by resolving the distinguished crossings shown in Figure 5 by $\searrow \rightarrow \asymp$ and $Y \rightarrow \check{ユ}$.

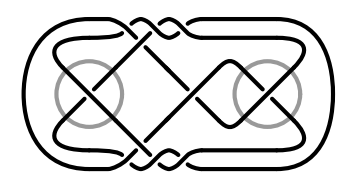

Figure 5: The distinguished crossings for the knot $10_{129}$.

The knot $K(\asymp ソ)=10_{137}$, so that the diagram of exact sequences (5) gives

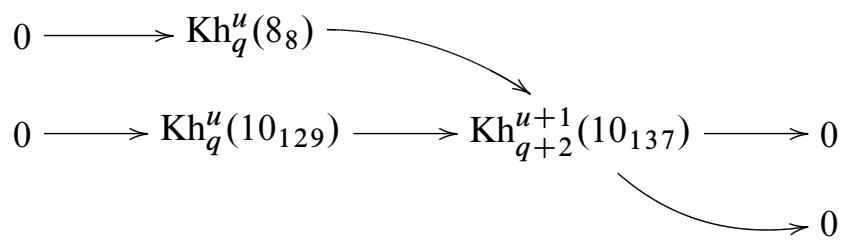

for $u>0$ and hence the isomorphism $\mathrm{Kh}_{q}^{u}\left(8_{8}\right) \cong \mathrm{Kh}_{q+2}^{u+1}\left(10_{137}\right) \cong \mathrm{Kh}_{q}^{u}\left(10_{129}\right)$ for $u>0$. Similarly, The knot $K(\searrow \smile)=8_{9}$ (this knot is shown in Figure 9), so that the 
diagram of exact sequences (8) gives

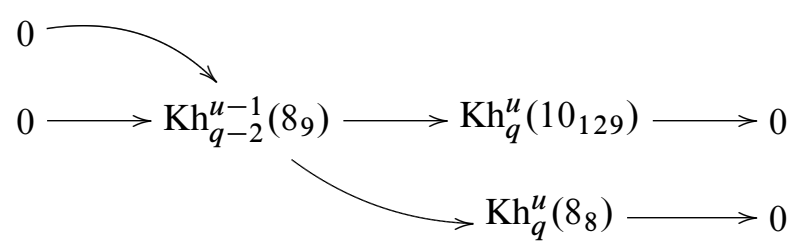

for $u<0$ and $\mathrm{Kh}_{q}^{u}\left(8_{8}\right) \cong \mathrm{Kh}_{q+2}^{u+1}\left(8_{9}\right) \cong \mathrm{Kh}_{q}^{u}\left(10_{129}\right)$ for $u<0$.

As in the proof of Lemma 3.1, the isomorphism in homological grading $u=0$ follows from Diagram (8) (for the torsion part) and Equation (1) and Equation (2) (for the free part).

Remark Note that this example illustrates the case of tangle connectivity of the form $\bigotimes$ for $T$ and $\bigotimes$ for $U$ in the proof of Lemma 3.1.

\subsection{Pairs of non-mutant prime knots}

To illustrate Theorem 4.1, we first need examples of prime simple tangles; these are provided in Figure 6. The fact that these are prime tangles for all $k \geq 0$ is shown by Lickorish [10, Section 2 Example (a)]. That these tangles are simple for $k \geq 0$ is an application of $k+2$ Reidemeister type II moves followed by a single Reidemeister type I move to see that $T+\emptyset$ is isotopic (fixing endpoints) to

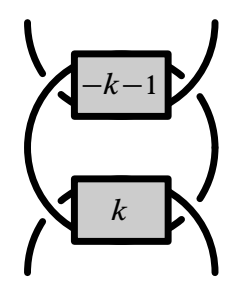

Figure 6: A prime simple tangle for $k \geq 0$.

The knots obtained from this construction in the case $k=0$ are shown in Figure 7.

Remark In the proof of Theorem 4.1, the fact that the knots obtained (for example, those of Figure 7) have distinct HOMFLYPT polynomials depends on the fact that $K_{\beta}(\Theta, \oslash)$ and $K_{\beta}(\bigotimes, \bigotimes)$ have distinct HOMFLYPT polynomial (cf [18, Theorem 1.1]). In the examples constructed for the proof Theorem 4.1 using $\beta=\sigma_{1}^{-1} \sigma_{2} \sigma_{1}^{-2}$, 

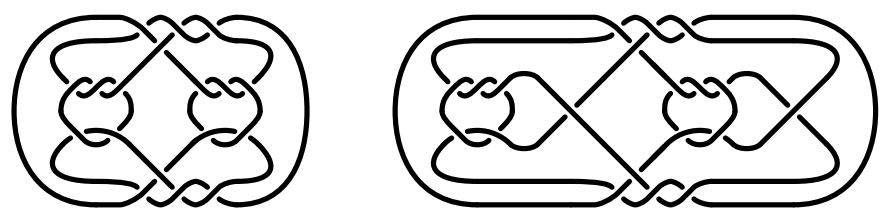

Figure 7: Non-mutant prime knots with identical Khovanov homology.

the knots are $4_{1} \# 4_{1}$ and $8_{9}$; these have distinct HOMFLYPT polynomials (cf Section 7.4). However any such pair will do, and many more examples exist; we give two to conclude this section.

If $\beta=\sigma_{1}^{-1} \sigma_{2} \sigma_{1}^{-3}$ then $K=K_{\beta}(\Theta, Q)=5_{2} \# 5_{2}^{\star}$ and we obtain $K^{\sigma}=10_{48}$. These have the same Khovanov homology by Lemma 3.1, while $K$ and $K^{\sigma}$ are distinguished by the HOMFLYPT polynomial.

If $\beta=\sigma_{1}^{-1} \sigma_{2} \sigma_{1}^{-1} \sigma_{2} \sigma_{1}^{-2}$ then $K=K_{\beta}(\vartheta, \vartheta)=6_{3} \# 6_{3}$ and we obtain $K^{\sigma}=12_{819}^{a}$. Again, these have the same Khovanov homology, while $K$ and $K^{\sigma}$ are distinguished by the HOMFLYPT polynomial.

Remark The reader may have observed that the base case in all of these examples is provided by taking a connected sum of a 2-bridge knot with its mirror image. While it is tempting to guess that any such connected sum will give rise to a family of examples, we leave it as an exercise to show that (at very least) the $(2, n)$-torus knots should be omitted since the action of $\sigma$ is trivial on these examples (consider $\beta=\sigma_{1}^{n}$ or $\left.\beta=\sigma_{1}^{-1} \sigma_{2}^{n-2} \sigma_{1}^{-1}\right)$.

\subsection{An aside on non-simple tangles}

It is natural to ask if Lemma 3.1 holds without the simplicity assumption on the tangles. For example, the knots $12_{990}^{a}$ and $12_{1225}^{a}$ arise in this way (consider the braid closure of $\beta \sigma_{2}^{3} \beta^{-1} \sigma_{2}^{-3}$ where $\left.\beta=\sigma_{1}^{-1} \sigma_{2} \sigma_{1}^{-2}\right)$. A second example of this phenomenon is given by the knots $12_{427}^{a}$ and $15_{45009}^{n}$ shown in Figure 8. Both of these examples share the same Khovanov homology (verified using the software KhoHo [16]). Indeed, as noted in Section 3, the simplicity requirement on the tangles is not required to show that knots obtained in this way have identical Jones polynomial [18]. However it seems optimistic (though tempting) to conjecture that Lemma 3.1 holds for all tangles.

Question Is there a knot $K_{\beta}(T, U)$ with non-simple tangles for which the action of $\sigma$ is detected by Khovanov homology? 
While the construction of Section 3 provides a wide range of examples of knots with identical Khovanov homology, the examples given in this section serve as a reminder that the restriction to simple tangles is a particularly special case.
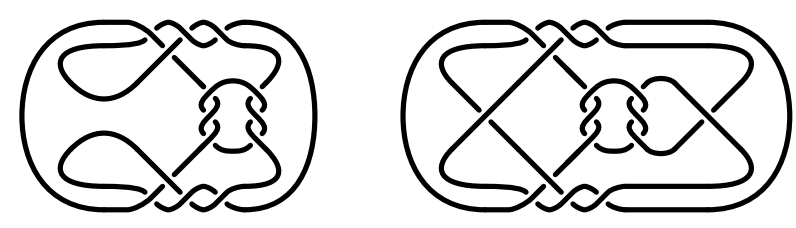

Figure 8: The knots $12_{427}^{a}$ and $15_{45009}^{n}$.

\subsection{Kanenobu's knots}

It has been shown that the action of $B_{3}$ may be iterated to obtain infinite families. We illustrate the case $n=1$ of Theorem 5.1 so that the braid in question is once more $\beta=\sigma_{1}^{-1} \sigma_{2} \sigma_{1}^{-2}$ to obtain a particular infinite family of knots with identical Khovanov homology. Let $K=K_{\beta}(\varangle, \bigotimes)$ so that $K^{\sigma}=K_{\beta}(\bigotimes, \bigotimes)$; the knots $K=4_{1} \# 4_{1}$, $K^{\sigma}=8_{9}$ and $K^{\sigma^{2}}=12_{462}^{n}$ are shown in Figure 9. By Lemma 3.1 these knots have the same Khovanov homology, while $4_{1} \# 4_{1}$ and $8_{9}$ (equivalently $8_{9}$ and $12_{462}^{n}$ ) have different HOMFLYPT polynomials. It should be noted that $4_{1} \# 4_{1}$ and $12_{462}^{n}$ share the same HOMFLYPT polynomial, and the interested reader should consult [5] in which Kanenobu originally classified this example. In particular, this provides an infinite family of distinct knots with homology $\mathrm{Kh}\left(8_{9}\right)$, and the case $n=1$ of Theorem 5.1.
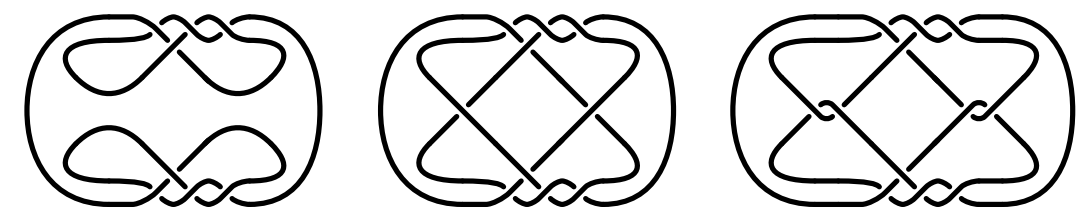

Figure 9: The first three knots in Kanenobu's sequence.

\subsection{Mutants}

The family of non-alternating knots with 13 crossings given in Figure 10 illustrate the construction used in the proof of Proposition 6.1. The four knots in question are 


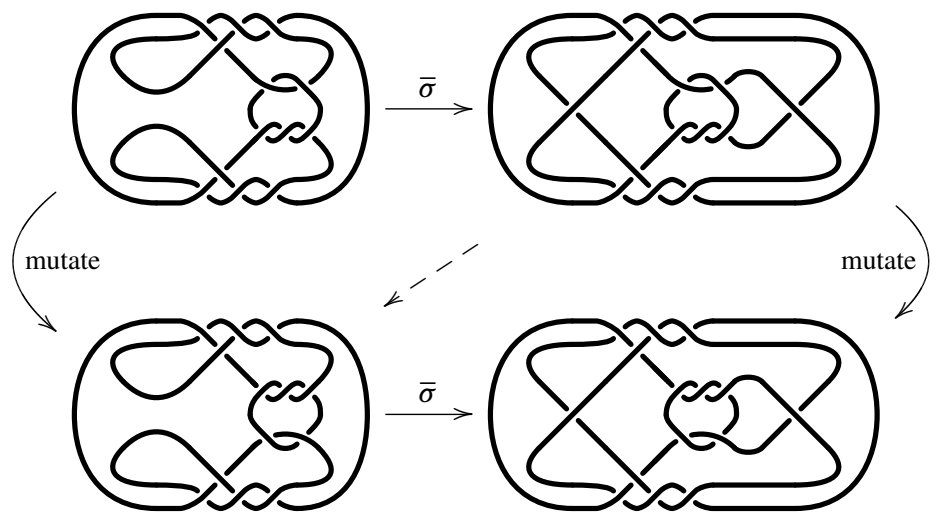

Figure 10: Two pairs of mutants illustrating Proposition 6.1.

arranged in Figure 10 so that pair in the first row $\left(13_{164}^{n}\right.$ and $\left.13_{922}^{n}\right)$ are related by twisting (and hence have identical Khovanov homology by Lemma 3.1), as are the pair in the second row $\left(13_{161}^{n}\right.$ and $\left.13_{795}^{n}\right)$. Note that we are acting by the inverse $\bar{\sigma}$ in this example. The columns are related by mutation (flipping across the horizontal axis), and the diagonal arrow in Figure 10 corresponds to the left action of $\sigma \in B_{2}$ used in the proof of Proposition 6.1, the second step of the mutation relating $13_{164}^{n}$ and $13_{161}^{n}$ (the knots in the left column of Figure 10). That is, each mutant pair (and indeed, any mutant pair of form given in Section 6) can be seen as the composition of right (twist) action, followed by a left (untwist) action.

\section{Acknowledgements}

Supported by a NSERC Canadian Graduate Scholarship.

\section{References}

[1] D Bar-Natan, Mutation Invariance of Khovanov Homology Available at http:// katlas.org/drorbn/

[2] D Bar-Natan, On Khovanov's categorification of the Jones polynomial, Algebr. Geom. Topol. 2 (2002) 337-370 MR1917056

[3] D Bar-Natan, Khovanov's homology for tangles and cobordisms, Geom. Topol. 9 (2005) 1443-1499 MR2174270

[4] D Bar-Natan, S Morrison, et al, The Knot Atlas Available at http://katlas.org/ 
[5] T Kanenobu, Infinitely many knots with the same polynomial invariant, Proc. Amer. Math. Soc. 97 (1986) 158-162 MR831406

[6] M Khovanov, Triply-graded link homology and Hochschild homology of Soergel bimodules arXiv:math.GT/0510265

[7] M Khovanov, A categorification of the Jones polynomial, Duke Math. J. 101 (2000) 359-426 MR1740682

[8] M Khovanov, L Rozansky, Matrix factorizations and link homology II arXiv: math.QA/0505056

[9] ES Lee, An endomorphism of the Khovanov invariant, Adv. Math. 197 (2005) 554-586 MR2173845

[10] W B R Lickorish, Prime knots and tangles, Trans. Amer. Math. Soc. 267 (1981) 321332 MR621991

[11] K Luse, Y Rong, Examples of knots with the same polynomials, J. Knot Theory Ramifications 15 (2006) 749-759 MR2253833

[12] J Rasmussen, Khovanov homology and the slice genus arXiv:math.GT/0402131

[13] J Rasmussen, Knot polynomials and knot homologies, from: "Geometry and topology of manifolds”, Fields Inst. Commun. 47, Amer. Math. Soc., Providence, RI (2005) 261-280 MR2189938

[14] D Rolfsen, Knots and links, Mathematics Lecture Series 7, Publish or Perish, Berkeley, CA (1976) MR0515288

[15] D Rolfsen, Global mutation of knots, J. Knot Theory Ramifications 3 (1994) 407-417 MR1291868

[16] A Shumakovitch, KhoHo - a program for computing and studying Khovanov homology Available at http://www.geometrie.ch/KhoHo/

[17] M Thistlethwaite, KNOTSCAPE - a knot theory computer Available at http:// wWw. math.utk.edu/ morwen/knotscape.html

[18] L Watson, Any tangle extends to non-mutant knots with the same Jones polynomial, J. Knot Theory Ramifications 15 (2006) 1153-1162 MR2287438

[19] S M Wehrli, Khovanov Homology and Conway Mutation arXiv:math.GT/0301312

Département de Mathématiques, Université du Québec à Montréal, Montréal, Canada, H3C 3P8

liam.watson@cirget.ca

http://www. cirget.uqam.ca/ liam/

Received: 9 January 2007 Revised: 5 August 2007 